\title{
How development of blood biomarkers could benefit prehospital management of acute stroke
}

\author{
Perttu J Lindsberg*,1,2, Markku Kuisma ${ }^{3}$ \& Olli S Mattila² \\ ${ }^{1}$ Department of Neurology, Helsinki University Hospital, Helsinki, Finland \\ ${ }^{2}$ Molecular Neurology, Research Programs Unit \& Clinical Neurosciences, University of Helsinki, Helsinki, Finland \\ ${ }^{3}$ Section of Emergency Medical Services, Department of Emergency Medicine, Helsinki University Hospital \& University of Helsinki, \\ Finland \\ * Author for correspondence: perttu.lindsberg@hus.fi \\ "What is clearly missing is an easier surrogate marker for recognizing the condition of brain \\ tissue"
}

First draft submitted: 25 July 2017; Accepted for publication: 15 August 2017; Published online:

28 November 2017

Keywords: biomarkers $\bullet$ emergency medical services $\bullet$ intracerebral hemorrhage $\bullet$ stroke $\bullet$ thrombectomy $\bullet$ thrombolytic therapy

Stroke is one of the leading causes of death worldwide and leaves most survivors with permanent disability [1,2]. Unfortunately, treatment options of acute stroke are still limited. In developed urban hospitals roughly a third of all ischemic stroke patients receive urgent recanalization therapy, in other words thrombolysis with recombinant tissue plasminogen activator (rtPA) and/or endovascular mechanical thrombectomy for large vessel occlusion (LVO). These treatments are highly efficacious and cost effective, but remain markedly underused [3]. Furthermore, their efficacy is time dependent, with strict therapeutic windows. To enhance the stroke chain of survival, current guidelines recommend a tiered system with acute stroke ready hospitals, primary stroke centers and comprehensive stroke centers to ensure timely initiation of intravenous rtPA with the least possible delay after symptom onset and rapid triage of large vessel occlusions eligible for thrombectomy in a comprehensive stroke center [3].

\section{The challenge of stroke diagnostics}

Time delay in the prehospital phase is the most important reason for missing recanalization therapy. Even for patients meeting the appropriate time window, the median prehospital delay is commonly around $2 \mathrm{~h}$ [4]. Emergency medical services (EMS) are challenged with the difficult task of identifying likely acute stroke from the wide range of acutely ill patients and ensuring rapid transport to the appropriate emergency department for immediate neurological examination and diagnostic brain imaging. For now, the main diagnostic groups causing acute strokelike symptoms, namely ischemic stroke, transient ischemic attack, hemorrhagic stroke and conditions mimicking stroke, can only be differentiated with hospital-based tests. EMS are therefore restricted to using simple, nonspecific clinical scales for stroke recognition, preventing them from initiating specific treatments.

Mobile CT-equipped ambulances, directed by an onboard stroke neurologist or telemedicine consultation, are one active approach to front load diagnostic and therapeutic procedures and reduce treatment delays [5,6], but require significant resources and are only applicable in urban settings, missing rural areas with longer transportation distances. What is clearly missing is an easier surrogate marker for recognizing the condition of brain tissue (either biochemical or functional) while the described, tiered prehospital stroke management is being advanced. Such markers, including blood troponin measurement and ECG recording, are widely used in the prehospital management of acute myocardial infarction, but are still unavailable for stroke. Useful point-of-care (POC) blood tests would have the significant benefit of being relatively inexpensive and more easily applicable around the world. 


\section{Rationale for stroke blood biomarkers}

What are the types of clinical questions where surrogate markers could guide individualized transportation and management?

- Did this patient just suffer a likely brain hemorrhage that requires rapid normalization of coagulation parameters and transport to a hospital with neurosurgical resources?

- Is this patient undergoing rapid loss of viable brain tissue due to cerebral ischemia? Is this a case of LVO requiring rapid thrombectomy at a comprehensive stroke center or rather a distal occlusion likely to respond to recombinant tissue plasminogen activator administered at the closest primary stroke center.

- What is the state of the blood-brain barrier (BBB), which protects the brain parenchyma from vasogenic edema and hemorrhagic complications? If this is a hemorrhagic stroke, will the patient suffer from imminent brain edema or secondary hematoma expansion during the first days after onset? If this is ischemic stroke, is acute recanalization therapy safe or is the patient at risk of symptomatic posthrombolytic intracerebral hemorrhage $(\mathrm{ICH})$ ? Should we prepare to treat elevating intracranial pressure due to brain edema or transfer to a hospital capable for hemicraniectomy?

- Is thrombolysis safe and pertinent despite symptoms started overnight or beyond accepted therapeutic time windows?

- What is the likely outcome of this patient?

At the tissue level, several central steps of stroke pathophysiology could be envisaged to be monitored by blood biomarkers. This could include enzyme activation, vasoactive mediators and liberated basal membrane components signaling early BBB degradation. As tissue disruption advances, release of constituents of endothelial, neuronal or glial cells and mediators of inflammatory cells could theoretically be used to identify the tissue response typical for either primary ischemic or hemorrhagic brain lesions. Leakage of these signals into the circulation is imminent through the disrupted BBB, but cerebrospinal fluid pathways may also be relevant. Within the luminal compartment, release of intermediates of blood coagulation and fibrinolysis may signal shifts in the pathways activated by blood clots and endogenous clot lysis. In addition to proteins, changes in inflammatory cell gene expression, brain-specific microvesicles and exosomes, free metabolites, lipids and free DNA might also represent suitable biomarker signals.

\section{Past research}

A long line of putative stroke biomarkers exists. Why has this not translated into clinically relevant applications? Likely factors include cessation of tissue circulation and the patent BBB, which may withold cerebral biomarker release from exceeding the detection threshold of conventional immunoassays. The systemic inflammatory and stress response following an acute cerebral event may not, within the first hours, differ significantly enough between the alternative illness groups to show relevant and specific differences. Lastly, almost all previous biomarker research has been performed in hospital cohorts, with samples collected after hospital admission, typically between 6 and $24 \mathrm{~h}$ of the postsymptomatic period. Such delayed sampling may unearth the earliest differences that may occur within the pivotal ultra-acute stage.

Previously identified stroke biomarker candidates have been reviewed $[7,8]$. Out of these markers, glial fibrillary acidic protein (GFAP) is so far the most promising for translation into prehospital use. In several hospital cohorts in which samples were collected upon hospital arrival, GFAP has systematically demonstrated high sensitivity and specificity for diagnosing acute ICH [9-11]. For example, Foerch et al. found sensitivity and specificity values of 84.2 and $96.3 \%$ respectively. Recently, Rozanski et al. extended these findings to the prehospital setting, collecting ambulance plasma samples from $25 \mathrm{ICH}$ and 49 ischemic stroke patients, and reporting a sensitivity and specificity of 36 and $100 \%$ for identifying ICH using a GFAP cutoff of $0.29 \mathrm{ng} / \mathrm{ml}$ [12]. The high specificity suggests that GFAP-based algorithms could be developed to initiate treatment in a significant portion of ICH patients, but larger studies are needed to determine optimal cutoff values for prehospital use.

\section{Future progress}

Despite the sluggish fulfillment of stroke biomarker development, the tide may be turning in view of recent technological advances. First, novel highly sensitive assays have emerged, most notably including the single molecule array (SIMOA), which has already shown significant promise in discovery of biomarkers for other neurological 
diseases [13,14]. Second, development of mass spectrometry instrumentation and applications has lowered the bar to optimize selected reaction monitoring assays for exploratory measurement of biomarker candidates, including brain specific proteins, without the need for laborious antibody production [15]. Third, advances in high-throughput proteomic tools now enable investigative screening of hundreds of proteins simultaneously in large cohorts [16,17]. Fourth and most important, for prehospital biomarkers, new large stroke cohorts have recently been recruited for dedicated discovery of prehospital biomarkers, with first blood samples obtained on scene, within the golden hour after symptom onset $[18,19]$. Finally, ongoing progress in the development of POC technology has provided a wide range of novel measurement techniques for development of rapid ambulance assays [20].

Risk stratification and individual treatment decisions in both stroke thrombolysis and invasive endovascular therapies are pressing clinical problems. Prehospital triage of acute stroke currently relies largely on estimating time delay from symptom onset, and pathophysiological tips reflecting tissue state and infarct evolution are not utilized on an individual level. The future scenario of having a rapid POC blood test to guide decisions already at the scene or immediately on hospital admission would be very attractive.

\section{Financial \& competing interests disclosure}

PJ Lindsberg is supported by Jane and Aatos Erkko foundation, governmental research funds of the Helsinki University Hospital, Sigrid Jusélius Foundation and Signe and Ane Gyllenberg foundation. OS Mattila is supported by grants from The Finnish Medical Foundation and The Finnish Foundation for Cardiovascular Research. The authors have no other relevant affiliations or financial involvement with any organization or entity with a financial interest in or financial conflict with the subject matter or materials discussed in the manuscript apart from those disclosed.

No writing assistance was utilized in the production of this manuscript.

\section{References}

1 GBD 2015 Mortality and Causes of Death Collaborators. Global, regional, and national life expectancy, all-cause mortality, and cause-specific mortality for 249 causes of death, 1980-2015: a systematic analysis for the Global Burden of Disease Study 2015. Lancet 388(10053), 1459-1544 (2016).

2 Feigin VL, Norrving B, Mensah GA. Global burden of stroke. Circulation Res. 120(3), 439-448 (2017).

3 Khandelwal P, Yavagal DR, Sacco RL. Acute ischemic stroke intervention. J. Am. Coll. Cardiol. 67(22), 2631-2644 (2016).

4 Puolakka T, Strbian D, Harve H, Kuisma M, Lindsberg PJ. Prehospital phase of the stroke chain of survival: a prospective observational study. J. Am. Heart Assoc. 5(5), pii:e002808 (2016).

5 Fassbender K, Balucani C, Walter S, Levine SR, Haass A, Grotta J. Streamlining of prehospital stroke management: the golden hour. Lancet Neurol. 12(6), 585-596 (2013).

6 Wu TC, Parker SA, Jagolino A et al. Telemedicine can replace the neurologist on a mobile stroke unit. Stroke 48(2), 493-496 (2017).

7 Kernagis DN, Laskowitz DT. Evolving role of biomarkers in acute cerebrovascular disease. Ann. Neurol. 71(3), 289-303 (2012).

8 Foerch C, Montaner J, Furie KL, Ning MM, Lo EH. Invited article: searching for oracles? Blood biomarkers in acute stroke. Neurology 73(5), 393-399 (2009).

9 Foerch C, Niessner M, Back T et al. Diagnostic accuracy of plasma glial fibrillary acidic protein for differentiating intracerebral hemorrhage and cerebral ischemia in patients with symptoms of acute stroke. Clin. Chem. 58(1), 237-245 (2012).

10 Luger S, Witsch J, Dietz A et al. Glial fibrillary acidic protein serum levels distinguish between intracerebral hemorrhage and cerebral ischemia in the early phase of stroke. Clin. Chem. 63(1), 377-385 (2017).

11 Ren C, Kobeissy F, Alawieh A et al. Assessment of serum UCH-L1 and GFAP in acute stroke patients. Sci. Rep. 6, 24588 (2016).

12 Rozanski M, Waldschmidt C, Kunz A et al. Glial fibrillary acidic protein for prehospital diagnosis of intracerebral hemorrhage. Cerebrovasc. Dis. 43(1-2), 76-81 (2016).

13 Andreasson U, Blennow K, Zetterberg H. Update on ultrasensitive technologies to facilitate research on blood biomarkers for central nervous system disorders. Alzh. Demen. 3, 98-102 (2016).

14 Bogoslovsky T, Wilson D, Chen Y et al. Increases of plasma levels of glial fibrillary acidic protein, tau, and amyloid $\beta$ up to 90 days after traumatic brain injury. J. Neurotrauma 34(1), 66-73 (2016).

15 Martínez-Morillo E, García Hernández P, Begcevic I et al. Identification of novel biomarkers of brain damage in patients with hemorrhagic stroke by integrating bioinformatics and mass spectrometry-based proteomics. J. Proteome Res. 13(2), 969-981 (2014).

16 Sattlecker M, Kiddle SJ, Newhouse S et al. Alzheimer's disease biomarker discovery using SOMA scan multiplexed protein technology. Alzh. Demen. 10(6), 724-734 (2014).

17 Lind L, Siegbahn A, Lindahl B, Stenemo M, Sundström J, Ärnlöv J. Discovery of new risk markers for ischemic stroke using a novel targeted proteomics chip. Stroke 46(12), 3340-3347 (2015). 


\section{Editorial Lindsberg, Kuisma \& Mattila}

18 Wendt M, Ebinger M, Kunz A et al. Copeptin levels in patients with acute ischemic stroke and stroke mimics. Stroke 46(9), 2426-2431 (2015).

19 Mattila OS, Harve H, Pihlasviita S et al. Ultra-acute diagnostics for stroke: Large-scale implementation of prehospital biomarker sampling. Acta Neurol. Scand. 136 (1), 17-23 (2017).

20 Song Y, Huang Y-Y, Liu X, Zhang X, Ferrari M, Qin L. Point-of-care technologies for molecular diagnostics using a drop of blood. Trends Biotechnol. 32(3), 132-139 (2014). 